\title{
Implementation of the 3D Edge Plasma Code EMC3- EIRENE on NSTX
}

\author{
J.D. Lore ${ }^{1,2}$, J.M. Canik ${ }^{2}$, Y. Feng ${ }^{3}$, J.-W. Ahn' ${ }^{2}$, R. Maingi², and V. Soukhanovskii ${ }^{4}$ \\ ${ }^{I}$ Oak Ridge Institute for Science and Education, Oak Ridge, TN 37831, USA \\ ${ }^{2}$ Oak Ridge National Laboratory, Oak Ridge, TN 37831, USA \\ ${ }^{3}$ Max-Planck-Institut für Plasmaphysik, Greifswald, Germany \\ ${ }^{4}$ Lawrence Livermore National Laboratory, Livermore, CA 94550, USA
}

Email: lorejd@ornl.gov

\begin{abstract}
The 3D edge transport code EMC3-EIRENE has been applied for the first time to the NSTX spherical tokamak. A new disconnected double null grid has been developed to allow the simulation of plasma where the radial separation of the inner and outer separatrix is less than characteristic widths (e.g. heat flux width) at the midplane. Modeling results are presented for both an axisymmetric case and a case where $3 \mathrm{D}$ magnetic field is applied in an $n=3$ configuration. In the vacuum approximation the perturbed field consists of a wide region of destroyed flux surfaces and helical lobes which are a mixture of long and short connection length field lines formed by the separatrix manifolds. This structure is reflected in coupled 3D plasma fluid (EMC3) and kinetic neutral particle (EIRENE) simulations. The helical lobes extending inside of the unperturbed separatrix are filled in by hot plasma from the core. The intersection of the lobes with the divertor results in a striated flux footprint pattern on the target plates. Profiles of divertor heat and particle fluxes are compared to experimental data, and possible sources of discrepancy are discussed.
\end{abstract}

\section{Introduction}

Non-axisymmetric fields affect both the transport and stability properties of tokamak plasmas. Even a small asymmetry in the ideal axisymmetric field, for example due to unavoidable field errors, can result in increased collisional transport [1], creation of magnetic islands [2], stochastic fields [3], and rotation damping [4]. 3D field structures are also generated intentionally by perturbing the plasma with control coils. Application of 3D fields can cause several effects that are of critical importance to future tokamaks such as ITER. ELMs are mitigated, suppressed, or triggered [5-9]. The pedestal profiles are affected, with some cases showing a flattening of the density and temperature profiles, while in others the pedestal pressure gradient increases. The heat and particle divertor loads are also affected, through strike point splitting [10-12] or changes in the divertor recycling properties, e.g., reattachment of the divertor plasma [13]. These effects can be seen as either detrimental behavior to be mitigated or as beneficial behavior to be exploited. With this viewpoint, the critical aspect is to have an understanding of the underlying physics so that reliable predictions can be made, and full advantage of potential benefits taken.

Modeling of the above effects is complicated due to the 3D geometry and the coupling of parallel and cross-field transport. The opportunity exists, however, to apply to tokamaks advanced 3D codes originally developed for stellarator research. The EMC3-EIRENE code [1416] is a coupled 3D Monte Carlo plasma fluid transport (EMC3) and kinetic neutral recycling and transport (EIRENE) package. The code includes transport of particles, electron and ion energy, and parallel momentum in stochastic fields with 3D plasma facing component geometries. EMC3-EIRENE has been applied to stellarators [15] and limiter tokamaks [17] and has recently 


\section{Implementation of EMC3-EIRENE on NSTX}

been extended to poloidally diverted tokamaks $[18,19]$. The code uses classical parallel transport coefficients and prescribed anomalous cross-field transport coefficients.

The primary purpose of this paper is to describe the implementation of the EMC3EIRENE code to the NSTX tokamak [20]. NSTX is a low aspect ratio device with a major radius of $0.85 \mathrm{~m}$ and a minor radius of $0.65 \mathrm{~m}$. Modeling is based on NSTX discharge $135183\left(\mathrm{~B}_{\mathrm{t}}=0.44 \mathrm{~T}\right.$, $\left.\mathrm{I}_{\mathrm{p}}=800 \mathrm{kA}, \mathrm{P}_{\mathrm{SOL}}=4 \mathrm{MW}, x=2.3, \delta=0.8\right)$. Figure 1 shows time traces from this shot. The axisymmetric equilibrium magnetic field is taken from $\mathrm{t}=433 \mathrm{~ms}$ (blue line in Fig. 1), after the application of the 3D fields, but before ELMs are triggered. This discharge exhibits clear strike point splitting in both the $\mathrm{D}_{\alpha}$ and heat flux measurements [11,13], both due to intrinsic error fields and applied perturbations. The perturbation field is applied in NSTX with a set of six approximately rectangular midplane coils external to the vacuum vessel, normally used for error field correction and resistive wall mode control [21]. The field can be applied in an $n=1,2$ or 3 configuration; all results shown here are for an $n=3$ field with a coil current $I_{c}$ of $1 \mathrm{kA}$. The spectrum of the applied field has strong resonant and non-resonant components [22].

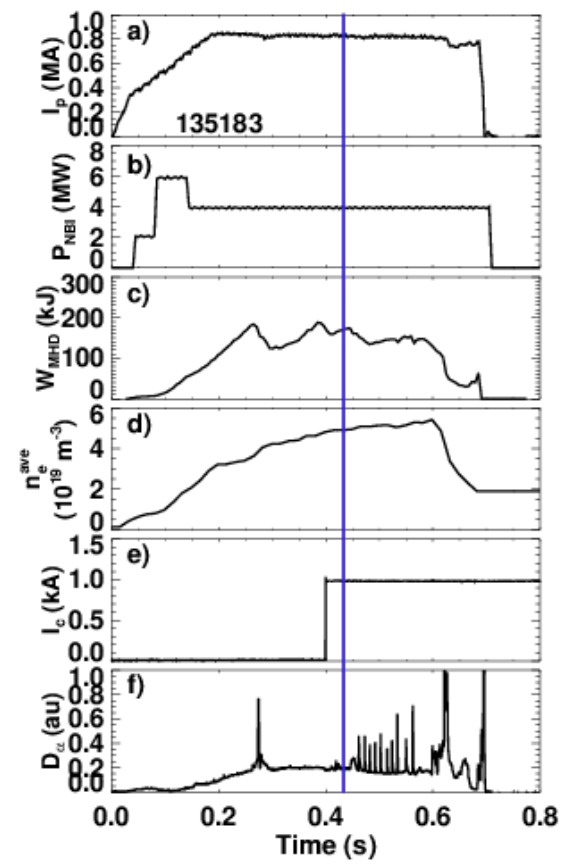

Figure 1. a) Plasma current, b) injected power, c) stored energy, d) line averaged electron density, e) perturbation coil current, and f) divertor $\mathrm{D}_{\alpha}$ light for shot 135183.

In order to perform modeling of discharge 135183, a disconnected double null grid has been implemented in EMC3-EIRENE for the first time. Results from two cases are presented, an axisymmetric configuration $\left(\mathrm{I}_{\mathrm{c}}=0 \mathrm{~A}\right)$ and one with the perturbation field applied using the vacuum approximation (see below). In the perturbed case, the magnetic field structure shows a wide region of destroyed flux surfaces with remnant islands, with intact flux surfaces only existing inside of $\psi_{\mathrm{N}} \approx 0.3$. Fieldlines followed from within the unperturbed separatrix intersect the targets in a series of helical lobes. These lobes, characteristic of the topological structures of dynamical systems known as homoclinic tangles, are formed by the splitting of the axisymmetric separatrix into multiple invariant manifolds $[23,24]$. The lobes are a mixture of long connection length stochastic field lines and short connection length 'laminar' field lines. The intersection of the lobes with the divertor target generates a striated pattern of strike point locations on the target face. This structure is reflected in the modeled temperature and density, resulting in striations in the divertor energy and particle fluxes. The initial modeling results are consistent with 


\section{Implementation of EMC3-EIRENE on NSTX}

measurements of the divertor fluxes, and patterns generated by fieldline following with and without an ideal plasma response [11,13].

Section 2 discusses details of the EMC3 grid, and the procedure used to generate the disconnected double null grid used for NSTX modeling. A description of the perturbed magnetic field structure is given in Section 3. Transport modeling using EMC3-EIRENE is presented in Section 4. Finally conclusions are given in Section 5.

\section{Generation of the EMC3 grid}

EMC3 modeling requires generation of a fully 3D computational grid. The code uses a fast, accurate reversible field line mapping (RFLM) [25] technique, which requires that the grid be field-aligned (i.e., incrementing the appropriate index starting from a grid point traces out the trajectory of a field line). This field alignment, which is necessitated by the strongly anisotropic transport, greatly increases the computational speed of the simulations, as direct field line integration is not required. On the other hand, the grid must be generated for each magnetic configuration of interest. The RFLM technique implemented in EMC3 has recently been advanced from a regularly structured grid to a block-structured grid [26]. This allows for a more efficient application of the code to configurations such as poloidally diverted tokamaks, which have clearly defined geometric regions ('blocks'). Each block is a full field aligned grid corresponding to a plasma region, for example 'core' and 'scrape-off layer' blocks can be defined, with the separatrix as the connecting boundary. Mapping is then performed at the interfaces between the blocks. In this way, appropriate radial and poloidal grid sizes can be chosen for each block, allowing for high resolution in the critical areas (e.g., near the targets and the x-point) and lower resolution in regions such as the core.

EMC3-EIRENE simulations have been performed for several poloidally diverted tokamak configurations, e.g., DIII-D [18] and ASDEX [19]. These simulations have been treated as single null, with three primary computational blocks: the core, the scrape-off layer (SOL), and the private flux region (PFR). Each of these blocks has a finite toroidal extent, and additional sets of the three primary blocks are generated until the total grid domain covers a periodic toroidal angle (e.g., $120^{\circ}$ for the application of $n=3$ perturbations discussed here). Outside of the main (plasma) computational grid additional grid cells are added for neutral particle calculations (see below).

Modeling of NSTX plasmas required the generation of a disconnected double null grid (see Fig. 2). Discharge 135183 has $\delta_{\mathrm{r}}^{\text {sep }} \sim 5 \mathrm{~mm}$ at the midplane, resulting in a large fraction of the input power lost through the boundaries of a lower single null grid (corresponding to the core=black, SOL=orange, PFR=blue cells in figure 2). Further, most of the lower horizontal target observed by the $\mathrm{D}_{\alpha}$ and fast IR cameras is not resolved (solid red horizontal line in Fig. 2b). To address these issues, three additional grid domains have been generated: upper PFR (upper purple region), high field side SOL (light blue), and low field side SOL (green). This grid allows for greater flexibility in the configuration modeled (including resolution of the upper targets), a larger radial extent at the midplane $\left(>\delta_{\mathrm{r}}^{\text {sep }}\right.$ ), and a computational domain capable of spanning the region of divertor measurement. 
Implementation of EMC3-EIRENE on NSTX
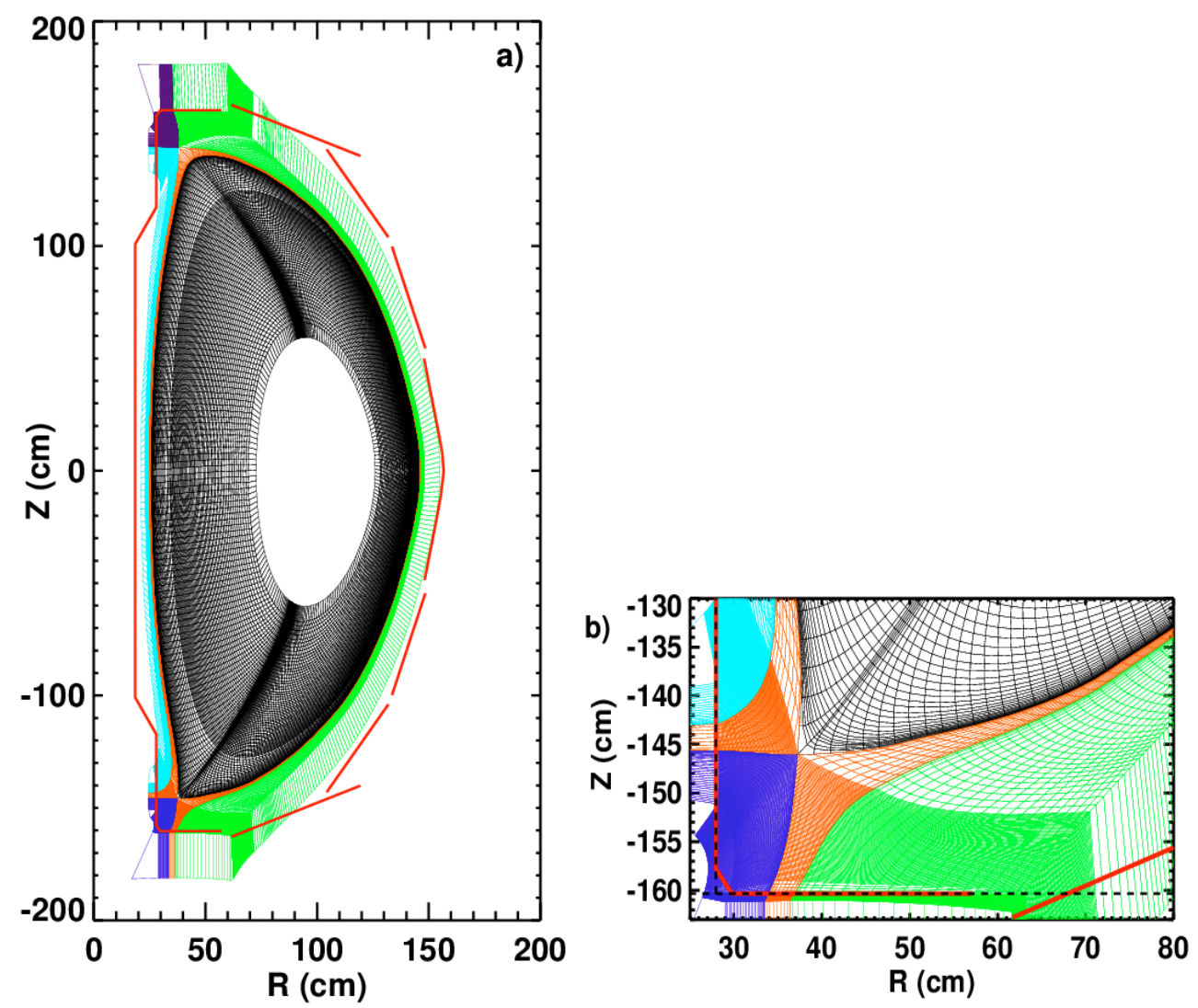

Figure 2. (a) Simple disconnected double null grid configuration for NSTX plasmas at $\phi=0^{\circ}$. (b) Lower divertor region showing increased resolution near the x-point and targets, and simplified divertor geometry used in modeling (dashed black lines).

The general method for generating the 3D grid consists of the following steps. First, each 2D grid block is generated in a given plane, typically at a fixed toroidal angle (Fig 2). Each block is extended into three dimensions by taking steps (in both directions) along fieldlines started from each of the 2D grid points. To satisfy the bilinear interpolation scheme of the RFLM technique used by EMC3, the cells must remain everywhere convex and have distortions that are approximately linear. These restrictions often limit the toroidal extent of a given grid block as the cells become increasingly deformed in regions of strong magnetic shear, particularly near the separatrix. When this occurs, an additional block is created from fieldlines started from a new 2D plane. The toroidal extent of the two blocks is chosen such that a common plane is shared, and a mapping surface is defined at this location. Care must also be taken that the $2 \mathrm{D}$ base grid cells are properly shaped in the regions around the x-points (Fig. 2b). This is achieved for NSTX grids through use of the Carre grid generator [27] provided with the SOLPS code package [28]. Carre attempts to produce orthogonal cells including efficient scaling of resolution (high resolution near $\mathrm{x}$-points and targets, lower resolution away from these areas). Use of a common grid generator also facilitates axisymmetric benchmarking of SOLPS and EMC3-EIRENE as a subset of the EMC3 grid can be used for SOLPS simulations. Axisymmetric benchmarking of NSTX plasmas (to be reported elsewhere) can be used to evaluate the impact of additional physics not included in the current version of EMC3, such as flux limiters [29].

The total computational grid defines a large number of 'geometric cells', which form the elementary units of the domain. For the results shown here the NSTX grid consists of approximately one million cells. These cells determine the 'metric' used to perform field line 


\section{Implementation of EMC3-EIRENE on NSTX}

interpolation, neutral particle tracing, and to define the local vectors for the Monte-Carlo calculations. To avoid memory issues, a subset of these cells are used to define a lower resolution physical mesh. These physical cells are used to store the results of the Monte-Carlo scoring for representing the plasma parameters. Boundary conditions are set at the edges of the physical mesh. For these simulations the inner boundary was set to $\psi_{\mathrm{N}} \approx 0.7$ with $\mathrm{n}=3 \times 10^{19} \mathrm{~m}^{-3}$ and $\mathrm{P}_{\mathrm{in}}=4 \mathrm{MW}$, corresponding to experimental conditions for discharge 135183. Here $\psi_{\mathrm{N}}(\psi$ $\left.\psi_{\text {sep }}\right) /\left(\psi_{\text {ax }}-\psi_{\text {sep }}\right)$, where $\psi_{\text {ax }}$ and $\psi_{\text {sep }}$ are the poloidal magnetic flux at the axis and the separatrix, respectively. Bohm sheath boundary conditions are used at the target plates, and temperature and density scrape off lengths of $3 \mathrm{~cm}$ at the outer radial boundaries. For proper application of the inner boundary condition the first radial set of grid points should lie on a closed (isothermal) flux surface to ensure domain closure. This can be problematic for plasmas with wide regions of stochasticity. Choosing an inner surface far inside the plasma implies high temperatures, meaning particles must be followed for a long distance for a fixed Monte-Carlo time step. The computation time is significantly increased, as many additional mapping calculations are performed. In future NSTX simulations where direct comparisons of radial profiles are made, the inner boundary must either be created far within the plasma $\left(\psi_{\mathrm{N}}<0.3\right.$, see Section 3$)$, or carefully chosen to lie on a remnant island chain or a "ghost surface" [30], where an isothermal surface is expected despite the existence of chaotic fields. Note that applying the code to the plasma core may violate assumptions appropriate for an edge fluid plasma model.

In addition to the main cells used for the plasma computation, extra cells are defined outside of the plasma domain for neutral particle calculations. The wall geometry has been simplified for NSTX simulations. Only the horizontal and vertical target plates near the upper and lower x-points are included as linear elements (compare solid red and dashed black lines in Fig. 2b). The targets are represented in the code by 'marking' geometric cells as opaque targets for charged particles. The plasma calculations are then performed using this discretized target, however the real target geometry is used for neutral transport. During post-processing the plasma quantities are mapped to the actual target geometry (see section 4).

\section{Magnetic field structure}

Computational grids have been constructed for both axisymmetric and perturbed NSTX plasmas. The perturbation fields were modeled using the vacuum approximation, where the vacuum applied fields are superimposed on the axisymmetric equilibrium field from EFIT [31]. It has been shown that the strike point locations from the vacuum approximation agree well with peaks in the divertor fluxes, and that these locations do not change significantly when an ideal plasma response is included [13]. These results indicate that a vacuum approach may be reasonable, at least for the edge plasma, however the effect of the plasma response on the transport has yet to be determined. Future EMC3-EIRENE simulations will incorporate various plasma response models to determine their effect.

Figure 3a shows a Poincare plot generated for the perturbed case by following fieldlines started inside of the unperturbed separatrix. With the application of 3D fields, a wide region of stochasticity is seen, with remnant islands near the core, and closed surfaces inside of $\psi_{\mathrm{N}} \approx 0.3$. The helical lobes formed by the separatrix manifolds intersect the upper and lower horizontal targets. Note the lobes shown in Fig. 3a are truncated by the boundaries of the plasma grid. It is anticipated that these lobes will guide hotter plasma from the pedestal to the targets, resulting in striations in the divertor flux footprint.

The magnetic fields structure can be visualized by calculating the wall-to-wall connection length, shown in Fig. 3 for the case of applied 3D fields. Figure 3a is generated by EMC3 by following fieldlines starting from each grid cell to a maximum length of $800 \mathrm{~m}$. Regions of short connection lengths extend around the 'edges' of the lobes, while longer connection length flux tubes in the 'middle' of the lobes connect the inner plasma to the target. The connection length is calculated on the lower horizontal divertor target by following fieldlines started along the target 


\section{Implementation of EMC3-EIRENE on NSTX}

face until they intersect another plasma facing component. As shown in Fig. 3b, clear 'fingers' are observed, corresponding to the intersection of the lobes with the target face.
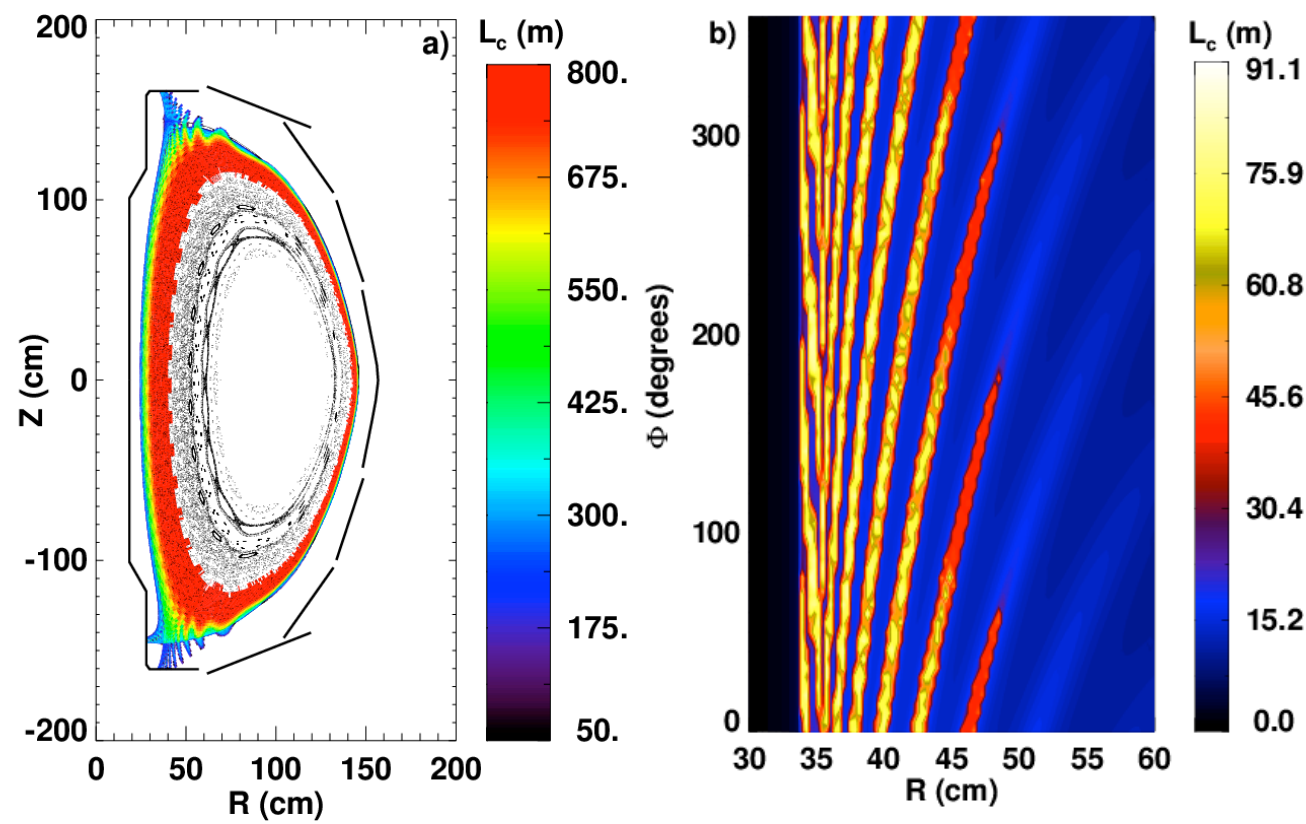

Figure 3. a) Wall-to-wall connection lengths in the R-Z plane at $\phi=0^{\circ}$ and b) at the lower horizontal target.

\section{Transport modeling}

Full transport calculations have been performed to determine the effects of the magnetic field structure on the plasma parameters. As mentioned above, EMC3 considers parallel transport as classical (Braginskii), with prescribed anomalous cross-field coefficients. For these simulations the anomalous transport coefficients were chosen to be $\mathrm{D}=0.24 \mathrm{~m}^{2} / \mathrm{s}$ and $\chi=2.5 \mathrm{~m}^{2} / \mathrm{s}$. These coefficients have not yet been varied to best match the measured radial profiles, but are similar to those used in previous 2D SOLPS modeling of NSTX discharges [32,33] with radially constant transport coefficients.

The modeled electron temperature for the axisymmetric and perturbed cases are shown in Figure 4. As anticipated, the underlying magnetic field structure is reflected in the transport quantities. Isothermal surfaces are now fully $3 \mathrm{D}$ at the plasma boundary in the perturbed case, with the lobe structure evident in the temperature contours. In the axisymmetric case the isothermal surfaces are the closed flux surfaces, as expected. 
Implementation of EMC3-EIRENE on NSTX
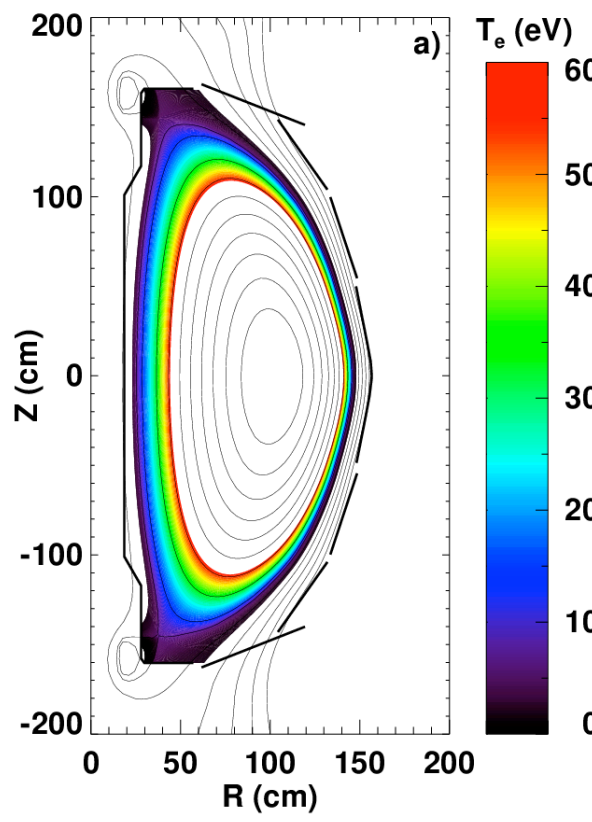

600.

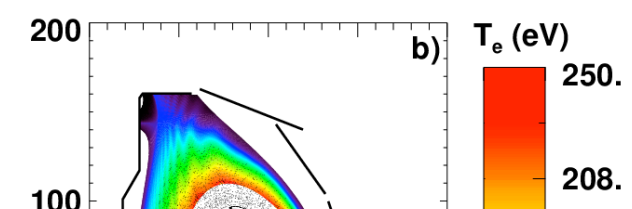

400.

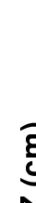

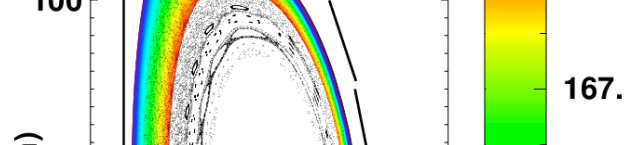

300.

$\frac{\text { E }}{\mathrm{N}} 0$

200.

100.

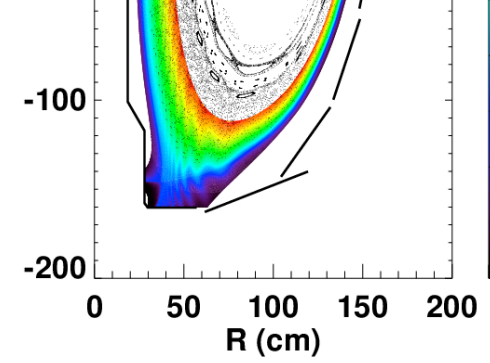

125.

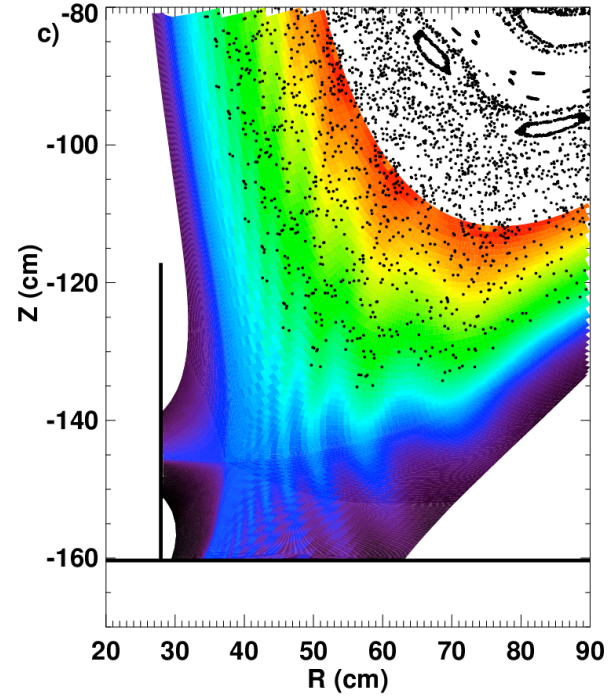

Figure 4. Electron temperatures at $\phi=0^{\circ}$ for a) axisymmetric and b) perturbed cases. c) zoom of the lower $\mathrm{x}$-point region of the perturbed case.

Figure 5 shows the energy and particle flux footprints for the perturbed case. Strike point splitting is observed, consistent with the connection length patterns of Fig. 3a. In this case the grid cells near the lower horizontal target have been constructed such that one edge of each cell intersecting the target lies exactly on the target face. This minimizes "shadowing" of the target face when mapping the plasma data stored in the discrete physical cells to the target face. However it should be noted that an axisymmetric target can never be perfectly represented due the field-aligned nature of the grid. 
Implementation of EMC3-EIRENE on NSTX

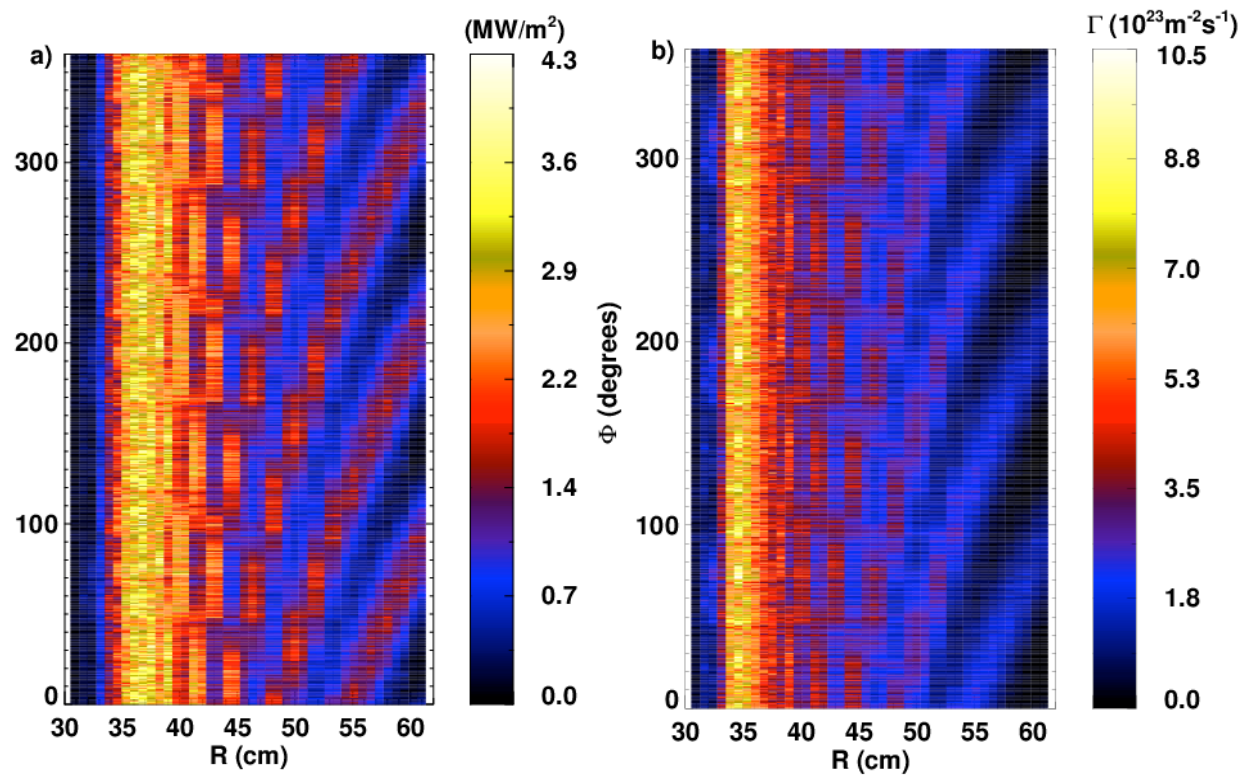

Figure 5. a) Energy flux and b) particle flux footprints on the lower horizontal target for the perturbed case

Normalized 1D radial profiles of the simulated target fluxes (solid black line) and measurements (dashed blue line) for an axisymmetric case are shown in Figure 6. The simulated heat flux is compared with uncalibrated measurements from an infrared (IR) camera; absolute calibration of the IR data is complicated by the divertor lithium coating present in these discharges. The simulated particle flux is compared to $\mathrm{D}_{\alpha}$ emission measurements from a fast CCD camera. Since the $\mathrm{D}_{\alpha}$ emission profile is not a direct substitution for particle flux, future comparisons will include simulated synthetic diagnostic comparisons of $\mathrm{D}_{\alpha}$ emission. While both the measured and modeled data are qualitatively similar, with a peak near the outer strike point (dot-dashed line) and a nearly monotonic decay, the radial positions of the peak fluxes and the decay lengths do not agree quantitatively. This preliminary comparison is subject to several sources of discrepancy which are discussed in this section and will be addressed in future work. While he radial positions of the peak values do not match exactly, the difference is on the order of the experimental uncertainty in the radial positions $(\sim 1-2 \mathrm{~cm})$. As both diagnostics are calibrated independently, relative shifts between the heat flux and $\mathrm{D}_{\alpha}$ profiles are possible. Further, the radial position of the profiles is sensitive to errors in the separatrix location as determined by the EFIT reconstruction. This error is estimated to be on the order of centimeters at the midplane, based on the electron temperature expected at the separatrix based on 2D edge modeling [32].

Given this uncertainty in the position of the target fluxes, and the relatively small shift required to align the maxima in the measured and simulated profiles, it is unclear whether the disagreement is indicative of plasma physics effects. On the other hand, plasma transport effects could also contribute to the observed differences. From the measured profiles, the maximum in the heat flux occurs at the separatrix location, while the peak in the $\mathrm{D}_{\alpha}$ profile is shifted slightly outward. This effect could be caused by different parallel transport regimes being important at different radii, e.g, if the plasma were sheath-limited near the separatrix and in the high-recycling regime farther out in radius (insufficient data exists to determine if this is the case in experiment). The modeled profiles, on the other hand, peak at approximately the same radial location, slightly outside of the strike point. In the model, the plasma is entirely in the conduction-limited regime under these conditions. Another factor that affects the comparison of experiment and modeling is the cross-field transport coefficients. In the modeling, these were not chosen to best match the midplane Thomson scattering profiles, (this would require spatially-varying coefficients to 


\section{Implementation of EMC3-EIRENE on NSTX}

reproduce the measured H-mode profiles [18]). Given the strong dependencies on target parameters on upstream density and temperature, it is to be expected that the target profiles in this case will not match exactly the measured profiles. For example, the modeled heat flux profile has a longer decay length as compared to the particle flux profile than is observed in the measured data (this is due to the large ratio of thermal to particle cross-field diffusivity). Improving the cross-field transport model to better match experiment will be a focus of future modeling efforts.

Figure 7 shows the normalized 1D flux profiles at the lower horizontal target for the perturbed plasma. In both the experimental and simulated data additional peaks can be seen corresponding to the lobes shown in Figure 5. The unperturbed strike point location is shown as a vertical dotdashed line for reference. However, the axisymmetric structure this corresponds to no longer exists in the presence of 3D fields (in the vacuum approximation), and the fluxes must not necessarily peak at this location. The measured and modeled heat flux profiles have a maximum at the same radial location, with agreement in the position of the additional peaks $(\mathrm{R} \approx 48$, $\mathrm{R} \approx 53 \mathrm{~cm})$. The modeled flux lacks the finer structure observed in the measured profile $(\mathrm{R}=40$ $44 \mathrm{~cm})$. This effect could be due to the high cross-field $\chi_{\mathrm{e}}$, which results in a smoothing out of the local peaks $[3,18]$ or the neglect of error fields, which are known to result in strike point splitting in NSTX [13]. Unlike the heat flux, the measured $D_{\alpha}$ profile is shifted outward by $\sim 2 \mathrm{~cm}$ as compared to the simulated particle flux profile. In addition to the sources of error discussed above, additional sources of uncertainty are present in the perturbed case. First, the perturbed field is modeled using a vacuum approximation, i.e., without plasma response. It is expected that the inclusion of plasma response would result in shielding of the perturbation field at rational surfaces, although amplification of perturbed fields is also possible. Second, as discussed above, the simulation and experiment may be in different parallel transport regimes with respect to the divertor plasma. Further, a transition in parallel transport regime versus radius can cause the relative shift of the simulated heat and particle flux profiles. Finally, the error in the toroidal position of the measured data should be accounted for in non-axisymmetric plasmas.
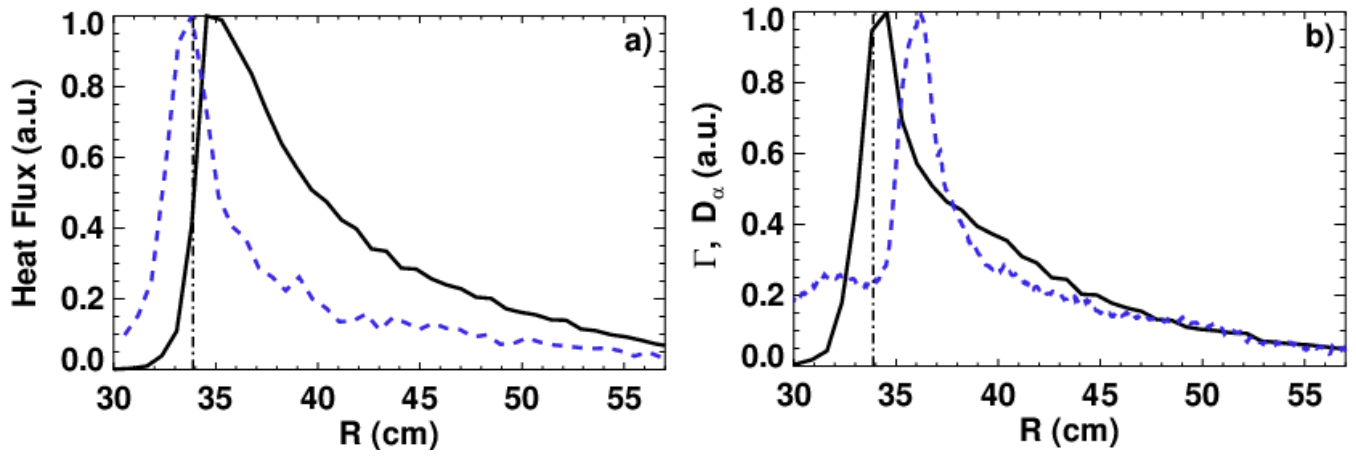

Figure 6. Modeled (solid black line) and measured (dashed blue line) normalized profiles of the a) heat flux and b) particle flux (model) and $\mathrm{D}_{\alpha}$ intensity (measurement) at the lower horizontal target for an axisymmetric plasma. Vertical dot-dashed line indicates the outer strike point of the unperturbed separatrix; the inner strike point intersects the vertical target. 
Implementation of EMC3-EIRENE on NSTX
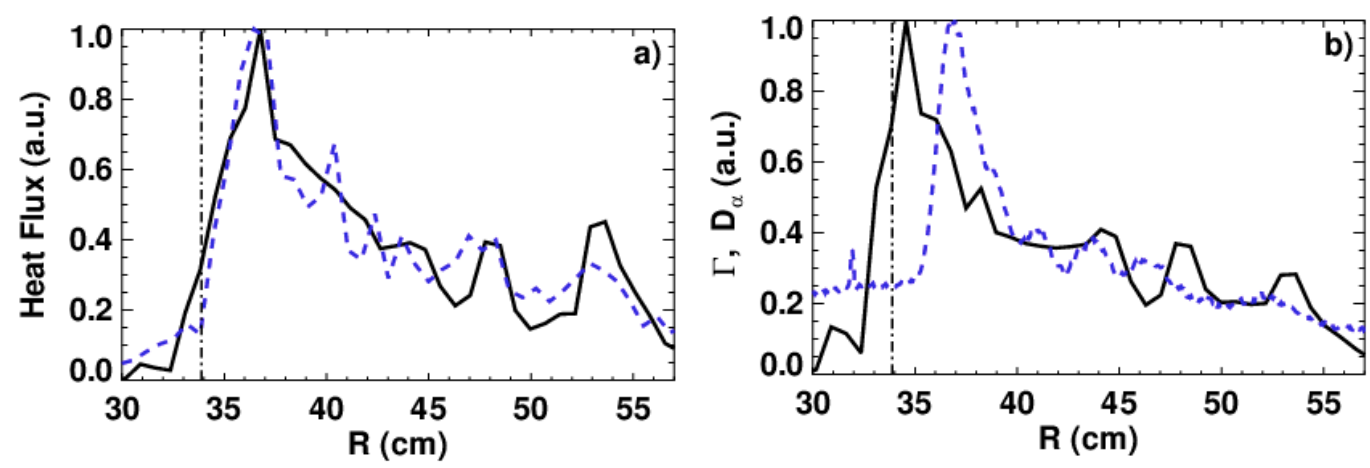

Figure 7. Modeled (solid black line) and measured (dashed blue line) normalized profiles of the a) heat flux and b) particle flux (model) and $\mathrm{D}_{\alpha}$ intensity (measurement) at the lower horizontal target for a perturbed plasma at $\phi=135^{\circ}$. Vertical dot-dashed line indicates the outer strike point of the unperturbed separatrix; the inner strike point intersects the vertical target.

\section{Conclusions}

The EMC3-EIRENE code has been applied for the first time to axisymmetric and perturbed NSTX plasmas. A new disconnected double null grid has been developed, in part using the Carre grid generator provided with SOLPS. Application of an $n=3$ field using the midplane coil set results in a stochastic magnetic field with a helical lobe structure created by the perturbed separatrix manifolds. This structure is reflected in EMC3-EIRENE simulations consisting of coupled 3D fluid transport, kinetic neutral particles, and recycling. Regions inside of the unperturbed separatrix have short connection lengths to the divertor, allowing hot plasma to fill the helical lobes. The intersection of these lobes with the target plates results in a striated pattern of fluxes, with clear strike point splitting found. Qualitatively, the modeled fluxes reproduce the trends found in the measured data: monotonically decreasing fluxes in the axisymmetric case, and additional peaks observed in the perturbed case corresponding to the intersection of the separatrix lobes with the target. Potential sources of error are discussed in section 4, with most differences consistent with a radial shift of the measured profiles on the order of the experimental uncertainties.

The axisymmetric simulations will next be benchmarked against the 2D SOLPS simulations. This allows for the evaluation of additional physics not included in the current version of EMC3-EIRENE, such as kinetic corrections. Future versions of the code will optionally include additional effects such as flux limiters, 3D varying anomalous transport coefficients, and pumping [34]. Future NSTX simulations will include the variation of cross-field coefficients to match the measured radial profiles, allowing for a direct comparison of divertor flux magnitudes, and additional observed effects such as the reattachment of the divertor plasma after the application of 3D fields with low gas puffing [35]. Additional field models, such as the inclusion of error fields and finite plasma response (e.g., an ideal plasma response from IPEC [36]) will also be implemented.

\section{Acknowledgements}

One author (J.D.L.) would like to thank Dr. T. Lunt and Dr. H. Frerichs for assistance in setting up the EMC3-EIRENE code and Dr. J.-K. Park for useful discussions. This work is supported in part by Magnetic Fusion Energy Postdoctoral fellowship from the Oak Ridge Institute for Science and Education and by U.S. Department of Energy Contract Numbers DE-AC05-00OR22725 and DE-AC02-09CH11466. 
Implementation of EMC3-EIRENE on NSTX

\section{References}

[1] Helander P. and Sigmar D., 2002 Collisional Transport in Magnetized Plasmas (Cambridge University Press, Cambridge UK)

[2] Fitzpatrick R., 1998 Phys. Plasmas 53325 (1998)

[3] Schmitz O., Evans T.E., Fenstermacher M.E., Frerichs H., Jakubowski M.W., Schaffer M.J., Wingen A., West W.P., Brooks N.H., Burrell K.H. et al 2008 Plasma Phys. Control Fusion 50 124029

[4] Shaing K.C., 2003 Phys. Plasmas 101443

[5] Shoji T., Tamai H., Miura Y., Mori M., Ogawa H., Leonard A.W., Jensen T., Hyatt A.W., Howald A.M., Fuchs G. et al 1992 J. Nucl. Mat. 196-198 296

[6] Fielding S.J., Buttery R.J., Field A.R., Jones P.B., Meyer H., Valovic M., Wilson H.R. and the COMPASS-D Physics and ECRH Teams 2001 Europhys. Conf. Abstr. 25A1825

[7] Evans T.E., Moyer R.A., Thomas P.R., Watkins J.G., Osborne T.H., Boedo J.A., Doyle E.J., Fenstermacher M.E., Finken K.H., Groebner R.J., et al 2004 Phys. Rev. Lett. 92235003

[8] Liang Y., Koslowski H.R., Thomas P.R., Nardon E., Alper B., Andrew P., Andrew Y., Arnoux G., Baranov Y. Bécoulet M., et al 2007 Phys. Rev. Lett. 98265004

[9] Canik J.M., Maingi R., Evans T.E., Bell R.E., Gerhardt S.P., LeBlanc B.P, Manikam J., Menard J.E., Osborne T.H., Park J.-K., et al 2010 Phys. Rev. Lett. 104045001

[10] Jakubowski M.W., Evans T.E., Fenstermacher M.E., Groth M., Lasnier C.J., Leonard A.W., Schmitz O., Watkins J.G., Eich T., Fundamenski W., et al 2009 Nucl. Fusion 095103

[11] Ahn J.-W., Canik J.M., Soukhanovskii V.A., Maingi R., and Battaglia D.J. 2010 Nucl. Fusion $\mathbf{5 0} 045010$

[12] Nardon E., Kirk A., Akers R., Bécoulet M., Cahyna P., Temmerman G. De., Dudson B., Hnat B., Liu Y.Q., Martin R., et al 2009 Plasma Phys. Control. Fusion 51124010

[13] Ahn J-W., Maingi R., Canik J.M., McLean A.G., Lore J.D., Park J-K., Soukhanovskii V.A., Gray T.K., and Roquemore A.L. 2011 Phys. Plasmas 18056108

[14] Feng Y., Sardei F., Kisslinger J., and Grigull P., 1997 J. Nucl. Mater. 241-243 930

[15] Feng Y., Sardei F., and Kisslinger J., 1999 J. Nucl. Mater. 266-269 812

[16] Reiter D., Baelmans M., and Börner P., 2005 Fusion Sci. Technol. 47172

[17] Kobayashi M., Feng Y., Sardei F., Reiter D., Finken K.H. and Reiser D. 2004 Nucl. Fusion 44 S64

[18] Frerichs H., Reiter D., Schmitz O., Evans T.E. and Feng Y., 2010 Nucl. Fusion 50034004

[19] Lunt T., Feng Y., Coster D., Wischmeier W., Wolfrum E., Fischer R., Müller H.W., Scarabosio A., Hicks N., and the ASDEX Upgrade team, Implementation and Application of EMC3-EIRENE at ASDEX Upgrade, 2009 36th EPS Conference on Plasma Physics (Sofia, Bulgaria) Vol 33E pp 1.154. http://epsppd.epfl.ch/Sofia/start.htm

[20] Ono M., Kaye S.M., Peng Y.-K.M., Barnes G., Blanchard W., Carter M.D., Chrzanowski J., Dudek L., Ewig R., Gates D. et al 2000 Nucl. Fusion 40557

[21] Sabbagh S.A., Bell R.E., Menard J.E., Gates D.A., Sontag A.C., Bialek J.M., LeBlanc B.P., Levinton F.M., Tritz K., and Yuh H., 2006 Phys. Rev. Lett. 97045004

[22] Canik J.M., Maingi R., Evans T.E., Bell R.E., Gerhardt S.P., Kugel H.W., LeBlanc B.P., Manickam J., Menard J.E., Osborne T.H. et al 2010 Nucl. Fusion 50034012

[23] Evans T.E. 2008 Implications of topological complexity and Hamiltonian chaos in the edge magnetic field of toroidal fusion plasmas Chaos, Complexity and Transport: Theory and Applications ed C. Chandre et al (Singapore: World Scientific)

[24] Sugiyama L.E. and Strauss H.R., 2010 Physics of Plasmas 17062505

[25] Feng Y., Sardei F., and Kisslinger J., 2005 Phys. Plasmas 052505

[26] Frerichs H., Reiter D., Feng Y., and Harting D. 2010 Comput. Phys. Commun. 18161

[27] Marchand R. and Dumberry M. 1996 Comput. Phys. Commun. 96232

[28] Schneider R., Reiter D., Zehrfeld H.P., Braams B., Baelmans M., Geiger J., Kastelewicz H., Neuhauser J. and Wunderlich R. 1992 J. Nucl. Mater. 196-198 810 


\section{Implementation of EMC3-EIRENE on NSTX}

[29] Malone R.C., McCrory R.L., and Morse R.L. 1975 Phys. Rev. Lett. 34721

[30] Hudson S.R. and Breslau J., 2008 Phys. Rev. Lett. 100095001

[31] Lao L.L., Ferron J.R., Groebner R.J., Howl W., St. John H., Strait E.J., and Taylor T.S. 1990 Nucl. Fusion 301035

[32] Canik J.M., Maingi R., Soukhanovskii V.A., Bell R.E., Kugel H.W., LeBlanc B.P. and Osborne T.H., 2011 J. Nucl. Mater. 415 S409.

[33] Canik J.M., Maingi R., Kubota S., Ren Y., Bell R.E., Callen J.D., Guttenfelder W., Kugel H.W., LeBlanc B.P., Osborne T.H., and Soukhanovskii V.A., 2011 Physics of Plasmas 18 056118

[34] Frerichs, H., et al, On gas flow effects in 3D edge transport simulations for DIII-D plasmas with resonant magnetic perturbations, Nuclear Fusion, accepted for publication. Part of the special issue associated with the 2011 Stochasticity in fusion plasma workshop.

[35] Ahn, J-W., Maingi R., McLean A.G., Canik J.M., Diallo A., Gray T.K., Jaworski M., LeBlanc B., Roquemore A.L., Soukhanovskii V.A., Tritz K, and Loarte A., Effect of 3-D fields

on divertor detachment and associated pedestal profiles in NSTX H-mode plasmas, $38^{\text {th }}$ EPS conference on plasma physics, P1.060 (2011).

http://ocs.ciemat.es/EPS2011PAP/html/contrib.html

[36] Park J.-K., Boozer A.H. and Glasser A.H. 2007 Phys. Plasmas 14052110 